\title{
Long-segment versus short-segment fixation through a posterior approach for tuberculous spondylodiscitis of the mid-thoracic spine in adults: a study of mid- to long-term efficacy
}

\section{Zhenchao Xu}

Xiangya Hospital Central South University

Xiyang Wang ( $\nabla$ wqlwqwxy@163.com )

Xiangya hospital https://orcid.org/0000-0003-1942-7517

\section{Gang Chen}

Xiangtan Central Hospital

Zheng Liu

Xiangya Hospital Central South University

\section{Research article}

Keywords: Long-segment fixation, short-segment fixation, posterior approach, tuberculous spondylodiscitis, mid-thoracic spine

Posted Date: August 25th, 2020

DOI: https://doi.org/10.21203/rs.3.rs-52923/v1

License: (a) (i) This work is licensed under a Creative Commons Attribution 4.0 International License. Read Full License

Version of Record: A version of this preprint was published at Journal of Neurosurgical Sciences on March 1st, 2021. See the published version at https://doi.org/10.23736/S0390-5616.21.05225-5. 


\section{Abstract \\ Background}

This retrospective study aimed to perform comparatively evaluate the mid- to long-term efficacy of longsegment and short-segment fixations via the posterior approach as treatment for tuberculous spondylodiscitis in the mid-thoracic spine.

\section{Methods}

A total of 95 patients with tuberculous spondylodiscitis in the mid-thoracic spine underwent surgery via the posterior approach including single-stage posterior debridement, interbody fusion, and pedicle screw fixation. Long-segment fixations were performed for 46 patients (group A), while short-segment fixations were performed for the other 49 patients (group B). Clinical and radiological outcomes were assessed during mid- to long-term follow-up.

\section{Results}

The average follow-up periods for groups $A$ and $B$ were $75.5 \pm 11.8$ and $76.8 \pm 11.6$ months, respectively. The operative time and intraoperative blood loss were lower in group $\mathrm{B}$ than in group $\mathrm{A}(P<0.05)$. Both management approaches significantly corrected the kyphotic deformity detected either in the early postoperative period or at the final visit after long-term follow-up $(P>0.05)$. Bony fusion was generated after average periods of $10.8 \pm 2.1$ months and $11.0 \pm 2.0$ months in groups $A$ and $B$, respectively. Favorable outcomes were observed on assessment of neurological function and patients' well-being at the final follow-up.

\section{Conclusion}

No therapeutic differences were observed between long-segment and short-segment fixation as surgical treatment for mid-thoracic Pott's disease during mid- to long-term follow-up. Kyphotic deformity and neurological impairment were significantly relieved via both posterior fixation approaches, with patients' well-being reaching a favorable level. Moreover, short-segment fixation led to less blood loss and required a shorter operative time.

\section{Background}

Tuberculous spondylodiscitis (Pott's disease), a common type of extrapulmonary tuberculosis (TB), is a main cause of kyphotic deformity and paraplegia [1]. Spinal TB is endemic in developing countries due to the poor health care systems and nonspecific manifestations in the early stage [2]. With the emergence of 
multi-drug resistant TB strains and HIV-triggered immunodeficiency diseases, the incidence of spinal TB in developed nations has shown an upward trend recently [3].

Young adults are commonly susceptible to tuberculous spondylodiscitis in the mid-thoracic (T5-T8) spine. High risks of paralysis are present due to the spread of TB into the spinal canal, resulting in compression-induced spinal cord ischemia [4]. Antituberculous drugs are employed in the management of TB. In addition, surgical intervention is required in severe cases to immediately correct kyphotic deformities and to restore the remaining biological function of the spinal cord $[5,6]$.

Surgical solutions have focused on the debridement of infected tissues that exhibit compromise, nerve decompression, and restoration of vertebral column stability by correction of kyphosis. The anterior approach was first reported in 1960 for successful removal of dead tissue and decompression of the spinal cord [7]. With this approach, a large incision in the thoracic tissues is needed to reach the infection site, increasing the surgical risks. Additionally, osteoporosis associated with infection results makes the vertebral structure fragile and may lead to instrument loosening or displacement [8, 9]. A combined anterior and posterior approach can overcome the stability-related disadvantage of the anterior approach, but this procedure is associated with more trauma and additional morbidity [10].

The posterior approach involves the use of a spinal instrumentation structure with screws and rods to correct the kyphotic deformity and decompress the spinal cord, facilitating treatment of tuberculous spondylodiscitis $[11,12]$. Pedicle screw fixation and a rod structure mechanically create a spinal curvature to prevent the progression of kyphotic deformity and spinal cord compression induced by wedging vertebrae. Two techniques for posterior screw fixation, long-segment and short-segment, are used in the surgical treatment. Short-segment fixation is applied on the vertebrae one-level apart from the lesion vertebra, while long-segment fixation involves two levels near the infected vertebra. Short-segment fixation requires a small incision but might have a high risk of failure due to high local stress on the fixation site. Long-segment can mechanically fix more vertebrae to develop a solid support for spinal column stabilization, but requires a large surgical incision. Studies comparing the two fixation techniques are lacking. To date, a comparative study evaluating the therapeutic benefits of these two fixations technique for Pott's disease has not been reported. The aim of the present study was to comparatively assess the mid- to long-term efficacies of the two fixation techniques for the treatment of tuberculous spondylodiscitis of the mid-thoracic spine.

\section{Methods}

\section{Patient data}

A total of 95 patients with tuberculous spondylodiscitis of the mid-thoracic spine were enrolled in this study from January 2010 to April 2015. The diagnosis of tuberculous spondylodiscitis was bases on clinical presentations, laboratory tests, and imaging examinations. Final confirmation was achieved by biopsy or tubercle bacillus culture. The indications for surgical treatment of TB lesions were as follows: 
involvement of less than two adjacent vertebrae, unpreventable progressive kyphosis, spinal cord compression, and weak efficacy observed for anti-TB medication. Patients with multi-segment tuberculous spondylodiscitis with severe destruction of vertebrae, severe osteoporosis, extensive cold abscess, history of congenital scoliosis, and ankylosing spondylitis were excluded. All patients received single-stage posterior debridement, interbody fusion, and long-segment fixation for group $A(n=46)$ or short-segment for group $B(n=49)$. Long-segment posterior fixation involved two adjacent vertebrae above and below the lesion vertebra, while short-segment fixation covered one adjacent vertebra on one side of the infected vertebra and two adjacent vertebrae on the other side.

The clinical symptoms included back pain, low fever, weight loss, and fatigue. Neurological impairment was present in some patients as well. All patients suffered thoracic back pain and/or intercostal neuralgia, and the pain intensity was assessed by the Visual Analog Scale (VAS). The erythrocyte sedimentation rate (ESR) and C-reactive protein (CRP) level exceeded the normal levels at the initial stage. Imaging examination approaches, including plain radiography, computed tomography (CT), and magnetic resonance imaging (MRI), were used to detect vertebral body collapse, bone destruction, kyphotic deformity, epidural and paravertebral abscess, and destructive intervertebral discs as well. The lesion location and preoperative characteristics of the patients are described in Fig. 1 and Table 1.

Table 1

Preoperative clinical characteristics of the included cases

\begin{tabular}{|llll|}
\hline & Group A & Group B & Statistical value \\
\hline Gender & $26 / 20$ & $27 / 22$ & - \\
\hline Age (years) & $46.7 \pm 14.4$ & $46.0 \pm 12.9$ & $P=0.765$ \\
\hline During of symptoms (months) & $3.7 \pm 1.9$ & $3.5 \pm 1.7$ & $P=0.731$ \\
\hline ESR $(\mathrm{mm} / \mathrm{h})$ & $57.3 \pm 20.0$ & $60.1 \pm 19.0$ & $P=0.237$ \\
\hline CRP $(\mathrm{mg} / \mathrm{L})$ & $42.5 \pm 9.9$ & $43.9 \pm 14.8$ & $P=0.631$ \\
\hline VAS & $7.0 \pm 1.1$ & $6.8 \pm 1.1$ & $P=0.327$ \\
\hline Kyphosis angle $\left(^{\circ}\right)$ & $22.2 \pm 2.8$ & $23.0 \pm 3.0$ & $P=0.231$ \\
\hline
\end{tabular}

\section{Preoperative preparation}

Standard anti-TB treatment was given to all patients for 2 weeks prior to surgery, including isoniazid (300 mg/day), rifampicin (450 mg/day), and pyrazinamide (750 mg/day), and ethambutol (750 mg/day). Nutritional support therapy was administered to resolve anemia and hypoproteinemia. Surgery was then performed when the ESR value returned to normal or dropped significantly. If a patient experienced acute paralysis or dramatic aggravation of neurological impairment during the anti-TB treatment, emergency surgery was required regardless of the ESR level.

\section{Surgical method}


In group A, pedicle screws were mounted on two adjacent vertebrae on each side of the infected vertebra (long-segment), and short pedicle screws were also fixed in the affected vertebrae if the pedicle screw channel was not destroyed. A temporary pre-bent rod was stabilized on the side where bone destruction or spinal neurological damage was relatively mild to maintain spinal stability during focal debridement. Two custom-fit rods as per the spinal curvature of a patient were longitudinally run through the screw heads to mechanically generate spinal stabilization and correct kyphosis. Autograft or allograft transplantation was employed for the intervertebral-associated intertransverse fusion. Streptomycin $(0.1 \mathrm{~g})$ and isoniazid $(0.3 \mathrm{~g})$ were used locally in the focus area, and the incision was closed in layers after placement of a drainage tube.

Group B received a short-segment solution involving screws and rods fixation one level apart from the infected vertebra on one side and two levels on the other side. All other procedures were the same as followed in group $A$.

\section{Postoperative management}

All patients received anti-TB regimens for 12-18 months after surgery. In addition, routine blood and hepatorenal function examinations as well as CRP level and ESR measurement were performed to evaluate efficacy. Rehabilitation training and physical therapy were performed as early as possible to prevent thrombus and improve neurological function. Patients were permitted to walk slowly with the assistance of a rigid thoracic-lumbar brace for 3 months. Regular re-examinations were required every 3 months in the first year postoperatively and every 6 months thereafter. Follow-up over 5 years was carried out for each patient to collect clinical and radiological data.

\section{Evaluating standards and statistical analysis}

Operation duration, intraoperative blood loss, and fusion period were documented for the two groups. Xray and CT examinations were performed to evaluate the internal fixation condition and bone grafting. The following assessment data were collected from the preoperative preparation to the end of follow-up: (1) ESR and CRP level; (2) VAS score; (3) SF-36 scale (36-Item short-form health survey questionnaire) scores for evaluating quality of life; (4) neurological assessment according to the American Spinal Injury Association (ASIA) impairment scale; and (5) kyphotic angles measured on lateral plain radiographs.

SPSS 20.0 software was used for statistical analysis. The follow-up outcomes of the two groups were compared by using Student's $t$ test. Deviation from a normal distribution was evaluated using the ranksum test. Differences were considered statistically significant if the $P$ value was less than 0.05 .

\section{Results}

\section{Clinical outcomes}

The postoperative patient data are summarized in Table 2. The average follow-up periods for groups $A$ and $B$ were $75.5 \pm 11.8$ and $76.8 \pm 11.6$ months, respectively. The operation duration and intraoperative 
blood loss in group A were greater than those in group $\mathrm{B}(P<0.05)$. The ESR and CRP values in the two groups returned to normal levels within 3 months postoperatively. No significant differences in the ESR and CRP values were found between the two groups at 3 months postoperatively or at final follow-up.

Table 2

Postoperative data of patients

\begin{tabular}{|c|c|c|c|}
\hline & Group A & Group B & Statistical value \\
\hline Follow-up period (months) & $75.5 \pm 11.8$ & $76.8 \pm 11.6$ & $P=0.647$ \\
\hline Operation period (min) & $192.4 \pm 27.6$ & $172.7 \pm 16.9$ & $P=0.000$ \\
\hline Intraoperative blood loss (ml) & $947.8 \pm 177.6$ & $810.2 \pm 165.8$ & $P=0.000$ \\
\hline \multicolumn{4}{|l|}{$\operatorname{ESR}(\mathrm{mm} / \mathrm{h})$} \\
\hline TMP & $11.1 \pm 3.1^{*}$ & $11.4 \pm 2.7^{\star}$ & $P=0.644$ \\
\hline FFU & $4.5 \pm 1.5^{\star}$ & $4.7 \pm 1.8^{*}$ & $P=0.668$ \\
\hline \multicolumn{4}{|l|}{ CRP (mg/L) } \\
\hline TMP & $4.3 \pm 1.3^{*}$ & $4.5 \pm 1.1^{*}$ & $P=0.578$ \\
\hline FFU & $1.8 \pm 0.7^{*}$ & $1.8 \pm 0.7^{*}$ & $P=0.860$ \\
\hline \multicolumn{4}{|l|}{ VAS } \\
\hline TMP & $2.0 \pm 0.8^{\star}$ & $1.9 \pm 0.8^{*}$ & $P=0.907$ \\
\hline FFU & $0.8 \pm 0.7^{\star}$ & $0.7 \pm 0.5^{*}$ & $P=0.522$ \\
\hline \multicolumn{4}{|l|}{ Kyphosis angle $\left(^{\circ}\right)$} \\
\hline Postoperative immediately & $12.3 \pm 1.4^{*}$ & $12.7 \pm 1.5^{\star}$ & $P=0.212$ \\
\hline FFU & $13.4 \pm 1.1^{*}$ & $13.8 \pm 1.5^{\star}$ & $P=0.075$ \\
\hline Correction rate (\%) & $44.2 \pm 4.9$ & $44.5 \pm 4.5$ & $P=0.931$ \\
\hline Correction loss $\left({ }^{\circ}\right)$ & $1.1 \pm 0.8$ & $1.1 \pm 0.7$ & $P=0.585$ \\
\hline Fusion period (months) & $10.8 \pm 2.1$ & $11.0 \pm 2.0$ & $P=0.772$ \\
\hline \multicolumn{4}{|c|}{ *Analyzed by paired t test, compared with preoperatively, $p<0.05$. } \\
\hline TMP three months postopera & FFU final fo & -up & \\
\hline
\end{tabular}

The mean VAS score was $7.0 \pm 1.1$ preoperatively and decreased to $0.8 \pm 0.7$ at the last follow-up in group A. Similarly, the mean VAS score of group B dropped to $0.7 \pm 0.5$ at the last visit from $6.8 \pm 1.1$ preoperatively. Patients' quality of life post-operation was significantly improved compared with that 
before operation in both groups $(P<0.05)$. At the final follow-up, there was no significant difference the SF-36 scale score between the two groups $(P>0.05$; Table 3$)$. 
Table 3

Quality of life according to SF-36 scale of the two groups

\begin{tabular}{|llll|}
\hline & Group A & Group B & Statistical value \\
\hline Physical Functioning & & & \\
\hline Preoperative & $64.1 \pm 5.7$ & $64.2 \pm 4.3$ & $P=0.911$ \\
\hline FFU & $79.8 \pm 3.1^{*}$ & $80.1 \pm 2.9^{*}$ & $P=0.726$ \\
\hline Role-Physical & & & \\
\hline Preoperative & $16.4 \pm 7.9$ & $16.8 \pm 8.6$ & $P=0.698$ \\
\hline FFU & $78.8 \pm 10.5^{*}$ & $80.2 \pm 11.8^{*}$ & $P=0.642$ \\
\hline Bodily Pain & & & \\
\hline Preoperative & $42.5 \pm 7.7$ & $43.4 \pm 7.4$ & $P=0.935$ \\
\hline FFU & $79.8 \pm 10.4^{\star}$ & $81.3 \pm 8.7^{*}$ & $P=0.441$ \\
\hline General Health & & & \\
\hline Preoperative & $24.6 \pm 7.9$ & $25.7 \pm 9.8$ & $P=0.627$ \\
\hline FFU & $76.8 \pm 8.1^{*}$ & $78.2 \pm 8.7^{*}$ & $P=0.509$ \\
\hline Vitality & & & \\
\hline Preoperative & $43.3 \pm 9.3$ & $42.2 \pm 7.8$ & $P=0.633$ \\
\hline FFU & $77.9 \pm 9.0^{*}$ & $77.6 \pm 9.4^{*}$ & $P=0.824$ \\
\hline Social Functioning & & & \\
\hline Preoperative & $24.1 \pm 9.7$ & $26.0 \pm 9.2$ & $P=0.409$ \\
\hline FFU & $75.2 \pm 8.1^{*}$ & $77.7 \pm 8.3^{*}$ & $P=0.136$ \\
\hline Role-Emotional & & & \\
\hline Preoperative & $28.4 \pm 9.5$ & $25.2 \pm 9.7$ & $P=0.104$ \\
\hline FFU & $78.5 \pm 8.6^{*}$ & $78.1 \pm 8.7^{*}$ & $P=0.757$ \\
\hline Mental Health & & & \\
\hline Preoperative & & & \\
\hline
\end{tabular}

*Analyzed by paired t test, compared with preoperatively, $p<0.05$.

FFU final follow-up 


\begin{tabular}{|c|c|c|c|}
\hline & Group A & Group B & Statistical value \\
\hline FFU & $77.8 \pm 8.9^{*}$ & $78.6 \pm 8.6^{*}$ & $P=0.781$ \\
\hline \multicolumn{4}{|c|}{ *Analyzed by paired t test, compared with preoperatively, $p<0.05$. } \\
\hline FFU final follow-up & & & \\
\hline
\end{tabular}

Neurological impairment was evaluated during the entire study from surgery to follow-up. The ASIA impairment scale protocol quantified spinal cord function in both groups at two stages, preoperative preparation and the last follow-up, as shown in Table 4. Increases in neurological function by at least one level were observed in $91.2 \%$ of patients in group $A$ and in $89.5 \%$ of patients in group B after surgery as per the ASIA impairment scale assessment.

Table 4

Neurological function according to the ASIA impairment scale of the two groups

\begin{tabular}{|llllll|}
\hline ASIA impairment scale & Group A & & & Group B \\
\cline { 2 - 3 } \cline { 5 - 6 } & Preoperative & FFU & & Preoperative & FFU \\
\hline A & 0 & 0 & & 0 & 0 \\
\hline B & 3 & 0 & 3 & 0 \\
\hline C & 9 & 0 & & 11 & 0 \\
\hline D & 22 & 11 & & 24 & 12 \\
\hline E & 12 & 35 & & 11 & 37 \\
\hline FFU final follow-up & & & & \\
\hline
\end{tabular}

\section{Radiographic outcomes}

In group $A$, the kyphosis angle was significantly corrected from $22.2 \pm 2.8^{\circ}$ preoperatively to $12.3 \pm 1.4^{\circ}$ immediately after surgery. The correction rate was $44.2 \pm 4.9 \%$, and the correction loss was $1.1 \pm 0.8^{\circ}$. The kyphosis angle in group B was significant decreased from $23.0 \pm 3.0^{\circ}$ preoperatively to $12.7 \pm 1.5^{\circ}$ immediately after surgery. The correction rate was $44.5 \pm 4.5 \%$, and the correction loss was $1.1 \pm 0.7^{\circ}$. No significant differences were recorded in the preoperative kyphosis angle, postoperative kyphosis angle, correction rate, and correction loss between the two groups $(P>0.05)$.

The average fusion periods were $10.8 \pm 2.1$ months and $11.0 \pm 2.0$ months in groups $A$ and $B$, respectively, with no significant difference between the two groups $(P>0.05)$. Surgical or postsurgical failures, such as nonunion, pseudoarthrosis, and loosening or fracture of instruments, were not detected at the last visit (Figs. 2 and 3).

\section{Complications}


No operative mortality or permanent neurological impairment occurred in either group. Five patients (three in group $A$ and two in group B) experienced superficial wounds that were cured by treatment with antibiotics. Local abscess recurrence due to irregular administration of anti-TB drugs was reported in two patients (one in group $A$ and one in group B), who were treated by catheter drainage through minimally invasive incision and regular chemotherapy. Seven patients (three in group A and four in group B) complained of postoperative intercostal neuralgia, which was managed using nonsteroidal antiinflammatory drugs.

\section{Discussion}

The mid-thoracic spine possesses excellent stability due to its restricted mobility and thorax support, and the majority of patients with mid-thoracic spinal TB can be cured with conservative treatment. Nevertheless, a longer duration of therapy with anti-TB drugs is required for patients with vertebral collapse, severe kyphotic deformity, or incurable cold abscess, which may bring about drug resistance [13]. Therefore, a therapeutic strategy based on standard pharmacotherapy combined with appropriate surgical treatment, which involves removal of the affected vertebrae and restoration of spinal stability, could accelerate the recovery of patients with tuberculous spondylodiscitis of the mid-thoracic spine [14].

Lesion removal, bone grafting, and internal fixation are regarded as critical techniques for surgical treatment of spinal TB. The posterior-only approach offers unique advantages for the treatment of tuberculous spondylodiscitis of the mid-thoracic spine. First, through the removal of the posterior column structure, this approach enables the affected vertebrae to be operated on with a direct view of the dura mater, and allows for simultaneous completion of bone grafting and correction of kyphotic deformity without spinal cord injury. Additionally, pedicle screws provide fixation of three columns, effectively restoring the normal physiological curvature of the spine, and thus, a better holding force that can reduce the risk of loosening and fracture of the grafts and provides formation a strong biological fixation in the temporary post-operation period. Intervertebral-associated intertransverse bone grafting to obtain $360^{\circ}$ fusion ensures long-term spinal stability. Furthermore, the posterior approach is less invasive and avoids damage to complex anatomical structures or vital anatomical organs. Therefore, many surgeons have adopted the posterior approach for treating mid-thoracic TB, which has achieved good curative effects [14-17]. The rationale for using the posterior approach is based on removal of the pathological tissues and the ossified bone from around the lesion that prevents the entry of anti-TB drugs, thus destroying the favorable living environment essential for the survival of Mycobacterium tuberculosis. The rest of the small amount of lesion and abscesses can be absorbed with postoperative standardized anti-TB chemotherapy [18]. In our series, the ESR and CRP level returned to normal within 3 months postoperation, and all patients had achieved solid bone fusion at the final follow-up. The results indicate that satisfactory curative effects were achieved with the posterior approach for tuberculous spondylodiscitis of the mid-thoracic spine.

Currently, there is no consensus regarding the best option for the fixed segment range of mid-thoracic spinal TB. TB patients who commonly have an impaired spinal column shape due to kyphotic deformity 
and a pinched spinal cord experience intense pain and mental suffering. A healthy spinal curvature rebuilt by a reasonable fixation of segments can significantly relieve back pain and improve the patient's quality of life. The long-segment fixation mechanically links more vertebrae (more than four) into the screw and rod structure to firmly support the upper trunk weight $[19,20]$. Body weight and extra mechanical loads are spread across more vertebrae by virtue of involvement of over four vertebrae [21-23]. An extra therapeutic benefit in the present study was the small correction angle loss over 5 years of follow-up with respect to an over $40 \%$ reduction of kyphotic deformity after surgery. However, the large incision needed for this fixation technique resulted in relatively high intraoperative blood loss and long surgical time. It should be noted that long-segment fixation created a rigid segment on thoracic spine and further caused a negative effect on adjacent joints degeneration. The rigid structure also restricted more spinal functional segments that were reported by patients during follow-up [20].

The clinical and imaging data from the mid- to long-term follow-up were also in favor of the specific strength of a short-segment fixation on the management of mid-thoracic spinal TB. The short-segment technique apparently retained more normal vertebrae with less trauma in surgery and preserved more spinal motion. Short-segment instrumentation was reported to have potential risks for inappropriately stabilizing the spine after removal of infected vertebral tissues, as well as for a high stress concentration in the fixation regions [24]. In this study, a short-segment employed one more level of vertebral fixation on one side of a lesion vertebra, with exactly two more pedicle screw fixations. This screw and rod structure created more supports to eliminate high local stresses [25]. In addition, sclerotic bone surrounding the affected vertebra might be helpful for the screw fixation thanks to its high bone density [26, 27]. As a result, no failure caused by screw loosening was reported in the short-segment group.

The mid- to long-term follow-up conducted in this study demonstrated that both long-segment and shortsegment fixations achieved the expected clinical goal: significantly reduced kyphotic deformities without failures of fixations such as loosened pedicle screws and cracked bone grafting. The kyphosis angle correction rate were $44.2 \pm 4.9 \%$ in group $A$ and $44.5 \pm 4.5 \%$ in group $B$. The correction losses were only $1.1 \pm 0.8^{\circ}$ and $1.1 \pm 0.7^{\circ}$ in groups $A$ and $B$, respectively, after 5 years of follow-up, suggesting a stable mechanical effect was achieved by those fixations. Moreover, the posterior approach-based surgical protocol promoted efficient intervertebral fusions, whereby stabilization of the spinal column was established. Those therapeutic benefits helped to restore a healthy spinal curvature, which positively reduced the health risks to the cardiopulmonary, neurological, and musculoskeletal systems. The VAS score reflecting pain intensity dropped over $80 \%$ over the long-term follow-up after surgical treatment. Significant increases in the SF-36 scores suggested favorable overall health status during follow-up. These assessments demonstrated that the patients' quality of life was improved, as expected.

Neither fixation technique was overwhelmingly superior in terms of long-term efficacy and health-related quality of life. Some published studies also have reported no significant difference between long-segment and short-segment fixations regarding clinical and radiological outcomes [28]. The comparative assessments in this study supported this finding based on a quantitative analysis of the correction of kyphotic deformities, VAS score for back pain, and SF-36 score for health status, especially mental health. 
The short-segment protocol was superior to the long-segment protocol with respect to less surgical blood loss and a shorter operation time. Nevertheless, the long-segment protocol was the only choice in specific cases with the following scenarios: severe kyphotic deformity, osteotomy involved in surgery, and osteoporosis. Therefore, neither segment protocol was found to be the overall best option for the posterior surgical approach to treating tuberculous spondylitis of the mid-thoracic spine.

This study also has some limitations. As a retrospective and single-center study with a relatively small sample size, statistical bias may have occurred. A multicenter study along with a large sample size is needed in the future to confirm the findings in this study and better characterize the benefits of the two approaches.

\section{Conclusions}

No therapeutic differences were observed between long-segment and short-segment fixations for surgical treatment of mid-thoracic Pott's disease over mid- to long-term follow-up. Kyphotic deformity and neurological impairment were significantly relieved via both posterior fixation approaches, and thus, patients' well-being reached to favorable levels. The short-segment fixation has strengths regarding surgical blood loss and operation duration compared with the long-segment approach, but was less suitable for the treatment of severe cases. In conclusion, neither segment technique was found to always be superior for the surgical treatment of TB.

\section{Abbreviations}

ASIA: American Spinal Injury Association; CRP:C-reactive protein; CT:computed tomography; MRI:magnetic resonance imaging; ESR:erythrocyte sedimentation rate; SF-36:Social Functioning-36; TB:tuberculosis; VAS:Visual Analogue Scale.

\section{Declarations}

\section{Ethics approval and consent to participate}

This study protocol was approved by the Ethics Committee of Xiangya Hospital, and written informed consent was obtained from all patients.

\section{Consent for publication}

All patients signed informed consent forms to publish their personal details in this article.

\section{Availability of data and materials}

The datasets and materials generated or analyzed during the current study are available from the corresponding author on reasonable request. 


\section{Competing interests}

The authors declare that they have no competing interests.

\section{Funding}

This work was supported by the National Natural Science Foundation of China (No. 81672191) and Xiangtan Science and Technology Project (SF-YB20181007). No benefit in any form has been or will be received from a commercial party related directly or indirectly to the subject of this manuscript.

\section{Authors' contributions}

ZC Xu participated in the design of this study, performed the statistical analysis, and drafted the manuscript. G Chen and Z Liu collected the clinical data and follow-up details of the study and conducted the study. XY Wang directed the study design and manuscript preparation. All authors read and approved the final manuscript.

\section{Acknowledgments}

Not applicable.

\section{References}

1. Jain AK, Kumar J. Tuberculosis of spine: neurological deficit. Eur Spine J. 2013;22(Suppl 4):624-33.

2. Hassan $\mathrm{K}$, Elmorshidy E. Anterior versus posterior approach in surgical treatment of tuberculous spondylodiscitis of thoracic and lumbar spine. Eur Spine J. 2016;25(4):1056-63.

3. Thakur K, Das M, Dooley KE, Gupta A. The Global Neurological Burden of Tuberculosis. Semin Neurol. 2018;38(2):226-37.

4. Li W, Liu Z, Xiao X, Zhang Z, Wang X. Comparison of anterior transthoracic debridement and fusion with posterior transpedicular debridement and fusion in the treatment of mid-thoracic spinal tuberculosis in adults. BMC Musculoskelet Disord. 2019;20(1):570.

5. Rajasekaran S, Shanmugasundaram TK, Prabhakar R, Dheenadhayalan J, Shetty AP, Shetty DK. Tuberculous lesions of the lumbosacral region. A 15-year follow-up of patients treated by ambulant chemotherapy. Spine (Phila Pa 1976). 1998;23(10):1163-7.

6. Moon MS, Moon YW, Moon JL, Kim SS, Sun DH. Conservative treatment of tuberculosis of the lumbar and lumbosacral spine. Clin Orthop Relat. 2002; (398): 40-49.

7. Hodgson A, Stock FE. Anterior spine fusion for the treatment of tuberculosis of the spine the operative findings and results of treatment in the first one hundred cases. $J$ Bone Joint Surg Am. 1960;42(2):295-310. 
8. Chiriano J, Abou-Zamzam AM Jr, Urayeneza O, Zhang WW, Cheng W. The role of the vascular surgeon in anterior retroperitoneal spine exposure: preservation of open surgical training. $\mathrm{J}$ Vasc Surg. 2009;50(1):148-51.

9. Luo C, Wang X, Wu P, Ge L, Zhang H, Hu J. Single-stage transpedicular decompression, debridement, posterior instrumentation, and fusion for thoracic tuberculosis with kyphosis and spinal cord compression in aged individuals. Spine J. 2016;16(2):154-62.

10. Wu W, Lyu J, Liu X, Luo F, Hou T, Zhou Q, Li Z, Chen Y, Li LT, Zheng Y, Wang G, Xu J, Zhang Z. Surgical Treatment of Thoracic Spinal Tuberculosis: A Multicenter Retrospective Study. World Neurosurg. 2018;110:e842-50.

11. Zeng H, Zhang P, Shen X, Luo C, Xu Z, Zhang Y, Liu Z, Wang X. One-stage posterior-only approach in surgical treatment of single-segment thoracic spinal tuberculosis with neurological deficits in adults: a retrospective study of 34 cases. BMC Musculoskelet Disord. 2015;16:186.

12. Wang $X$, Pang $X$, Wu $P$, Luo $C$, Shen $X$. One-stage anterior debridement, bone grafting and posterior instrumentation vs. single posterior debridement, bone grafting, and instrumentation for the treatment of thoracic and lumbar spinal tuberculosis. Eur Spine J. 2014;23(4):830-7.

13. Wang Z, Shi J, Geng G, Qiu H. Ultra-short-course chemotherapy for spinal tuberculosis: five years of observation. Eur Spine J. 2013;22(2):274-81.

14. Wu P, Wang XY, Li XG, Shen XJ, Pang XY, Luo CK, Xu ZQ, Zeng H, Zhang PH, Peng W. One-stage posterior procedure in treating active thoracic spinal tuberculosis: a retrospective study. Eur $\mathrm{J}$ Trauma Emerg Surg. 2015;41(2):189-97.

15. Wu P, Luo C, Pang X, Xu Z, Zeng H, Wang X. Surgical treatment of thoracic spinal tuberculosis with adjacent segments lesion via one-stage transpedicular debridement, posterior instrumentation and combined interbody and posterior fusion, a clinical study. Arch Orthop Trauma Surg. 2013;133(10):1341-50.

16. Zhou Y, Li W, Liu J, Gong L, Luo J. Comparison of single posterior debridement, bone grafting and instrumentation with single-stage anterior debridement, bone grafting and posterior instrumentation in the treatment of thoracic and thoracolumbar spinal tuberculosis. BMC Surg. 2018;18(1):71.

17. Du X, Ou YS, Zhu Y, Zhao ZH, Luo W, He B, Peng QQ, Hu JY. One stage posterior debridement, nonstructural bone graft in the surgical treatment of single segment thoracic tuberculosis: $\mathrm{A}$ retrospective single-center cohort study. Int J Surg. 2019;65:134-9.

18. Xu Z, Wang X, Shen X, Luo C, Zeng H, Zhang P, Peng W. Posterior only versus combined posterior and anterior approaches for lower lumbar tuberculous spondylitis with neurological deficits in the aged. Spinal cord. 2015;53(6):482-7.

19. Liu Z, Zhang P, Zeng H, Xu Z, Wang X. A comparative study of single-stage transpedicular debridement, fusion, and posterior long-segment versus short-segment fixation for the treatment of thoracolumbar spinal tuberculosis in adults: minimum five year follow-up outcomes. Int Orthop. 2018;42(8):1883-90. 
20. Altay M, Ozkurt B, Aktekin CN, Ozturk AM, Dogan O, Tabak AY. Treatment of unstable thoracolumbar junction burst fractures with short- or long-segment posterior fixation in magerl type a fractures. Eur Spine J. 2007;16(8):1145-55.

21. Jain AK, Jain S. Instrumented stabilization in spinal tuberculosis. Int Orthop. 2012;36(2):285-92.

22. Basaran R, Efendioglu M, Kaksi M, Celik T, Mutlu I, Ucar M. Finite Element Analysis of Short- Versus Long-Segment Posterior Fixation for Thoracolumbar Burst Fracture. World Neurosurg. 2019;128:e1109-17.

23. Liao JC, Chen WP, Wang H. Treatment of Thoracolumbar Burst Fractures by Short-Segment Pedicle Screw Fixation Using a Combination of Two Additional Pedicle Screws and Vertebroplasty at the Level of the Fracture: A Finite Element Analysis. BMC Musculoskelet Disord. 2017;18(1):262.

24. Dick JC, Jones MP, Zdeblick TA, Kunz DN, Horton WC. A biomechanical comparison evaluating the use of intermediate screws and cross-linkage in lumbar pedicle fixation. J Spinal Disord. 1994;7(5):402-7.

25. Wood KB, Li W, Lebl DR, LebI DS, Ploumis A. Management of thoracolumbar spine fractures. Spine J. 2014;14(1):145-64.

26. Xu Z, Wang X, Wu P, Pang X, Luo C, Zhang P, Zeng H, Peng W. Surgical treatment for mono-segmental lumbar tuberculosis by single-stage posterior debridement, compact bone grafting and posterior single-segment fixation. Injury. 2015;46(7):1311-6.

27. Liu Z, Li W, Xu Z, Wang X, Zeng H. One-stage posterior debridement, bone grafting fusion, and monosegment vs. short-segment fixation for single-segment lumbar spinal tuberculosis: minimum 5-year follow-up outcomes. BMC Musculoskelet Disord. 2020;21(1):86.

28. Aly TA. Short Segment versus Long Segment Pedicle Screws Fixation in Management of Thoracolumbar Burst Fractures: Meta-Analysis. Asian Spine J. 2017;11(1):150-60.

\section{Figures}




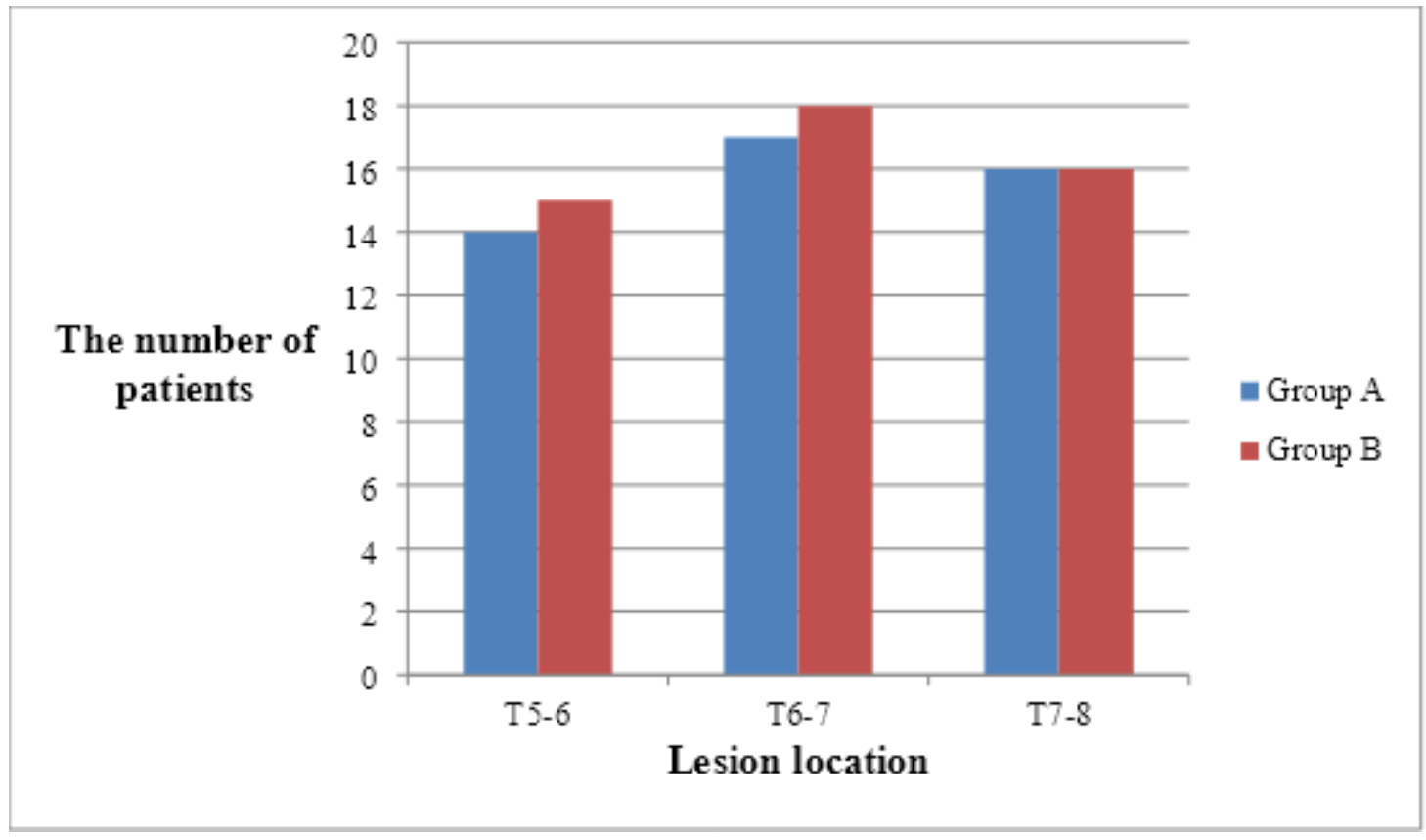

Figure 1

Segments affected by lesions
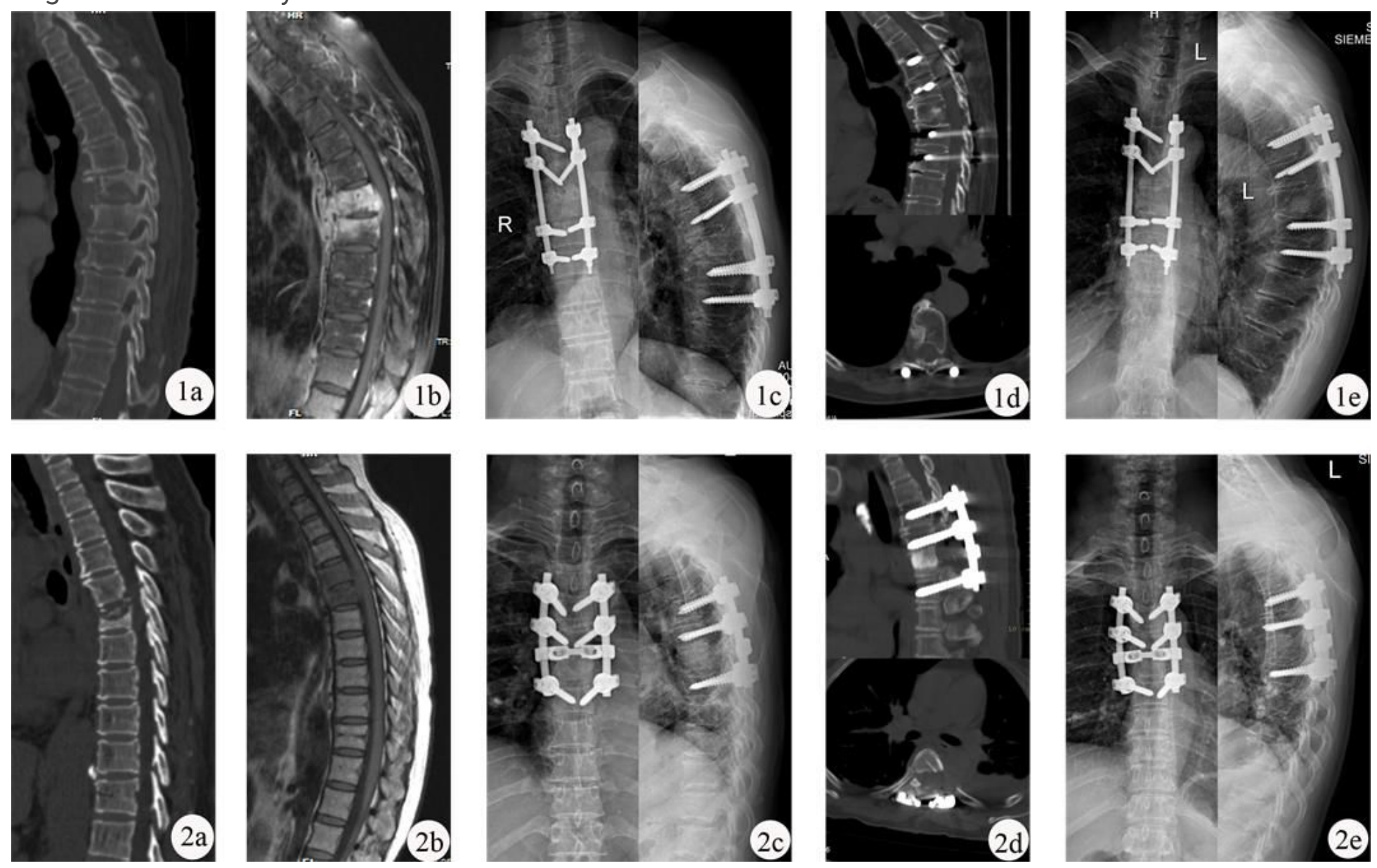

Figure 2 
A 61-year-old female with T5-L6 TB received one-stage posterior debridement, interbody fusion, and long-segment fixation. (1a-1b) Preoperative images showing vertebral destruction with deformity (kyphosis angle $27^{\circ}$ ) and spinal cord compression. (1c) Postoperative X-ray demonstrating the correction of the deformity (kyphosis angle was 15 ). (1d) CT images showing satisfactory bone fusion at 12 months. (1e) X-ray images displaying good internal fixation position and solid bone fusion, with no loss of correction after a follow-up period of 69 months. A 49-year-old female with T5-L6 TB received onestage posterior debridement, interbody fusion, and short-segment fixation. (2a-2b) Preoperative images showing lesions with a kyphosis angle of $19^{\circ}$. (2c) Postoperative X-ray demonstrating the correction of the deformity (kyphosis angle $10^{\circ}$ ). (2d) CT images showing satisfactory bone fusion at 9 months. (2e) X-ray images displaying good internal fixation position and solid bone fusion, with a kyphosis angle of $11^{\circ}$ after the follow-up period of 72 months.
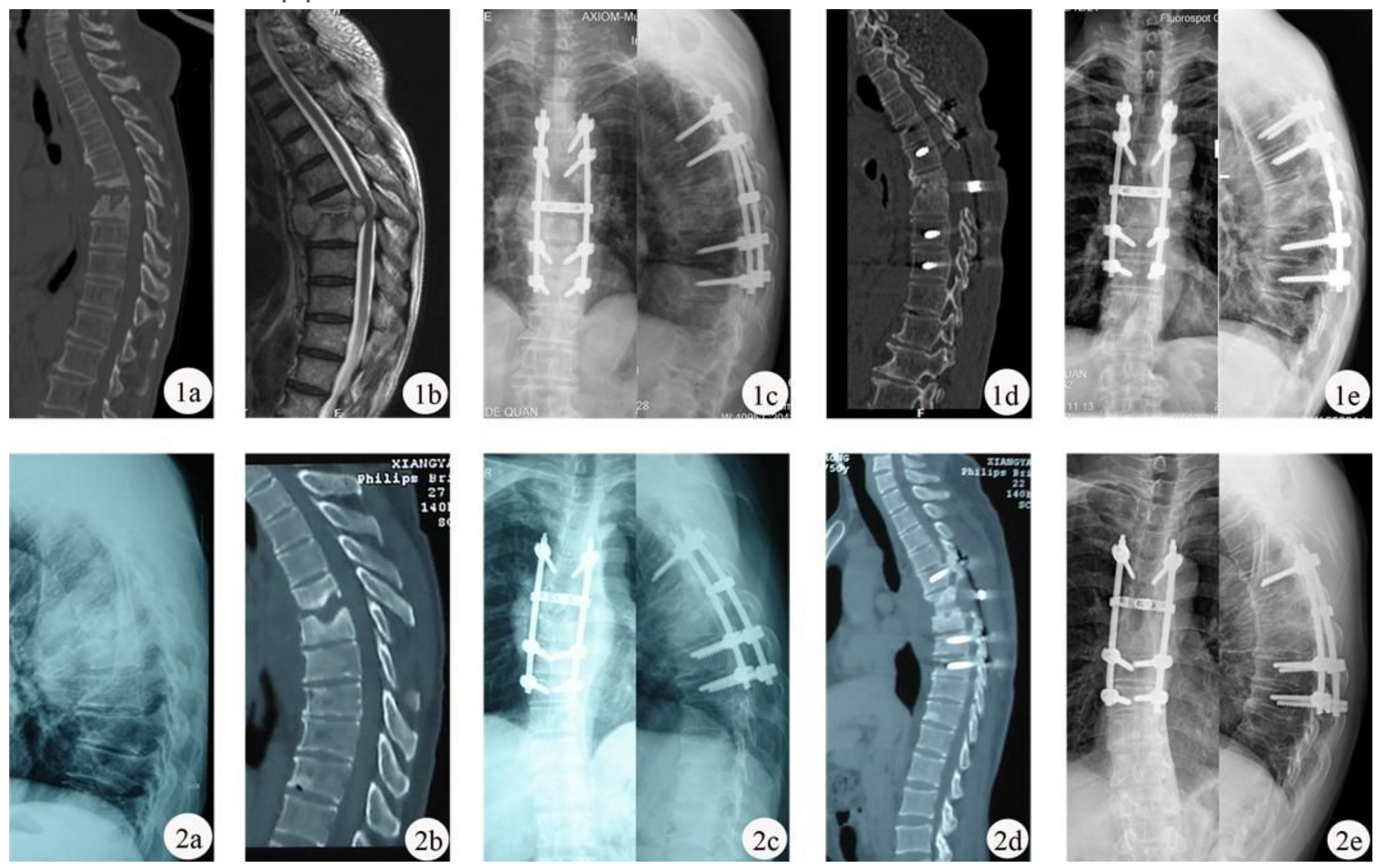

\section{Figure 3}

A 63-year-old male who was diagnosed as T6-L7 TB received one-stage posterior debridement, interbody fusion, and long-segment fixation. (1a-1b) Preoperative images showing vertebral collapse and abscess formation, with a kyphosis angle $28^{\circ}$. (1c) Postoperative X-ray demonstrating the correction of the deformity (kyphosis angle 13 ${ }^{\circ}$ ). (1d) CT showing compete bone fusion at 12 months. (1e) X-ray displaying good internal fixation position and solid bone fusion, with a correction loss of $1^{\circ}$ after the follow-up period of 81 months. A 50-year-old male who was diagnosed as T6-L7 TB received one-stage posterior debridement, interbody fusion, and short-segment fixation. (2a-2b) Preoperative images 
showing vertebral destruction with a kyphosis angle $26^{\circ}$. (2c) Postoperative X-ray demonstrating the correction of the deformity (kyphosis angle $14^{\circ}$ ). (1d) CT showing satisfactory bone fusion was obtained at 12 months. (2e) X-ray images displaying good internal fixation position and solid bone fusion, with no loss of correction after the follow-up period of 63 months. 